\title{
Impact of cocoa polyphenol extracts on the immune system and microbiota in two strains of young rats
}

\author{
Malen Massot-Cladera, Mar Abril-Gil, Sandra Torres, Àngels Franch, Margarida Castell and \\ Francisco J. Pérez-Cano* \\ Departament de Fisiologia, Facultat de Farmàcia, Institut de Recerca en Nutrició i Seguretat Alimentària (INSA-UB), \\ Universitat de Barcelona, Barcelona, Spain
}

(Submitted 10 April 2014 - Final revision received 4 August 2014 - Accepted 26 August 2014 - First published online 27 October 2014)

\begin{abstract}
A diet containing $10 \%$ cocoa, a rich source of polyphenols and fibre, is able to modify intestinal immune status as well as microbiota composition. The present study was aimed at investigating whether cocoa flavonoid content is uniquely responsible for these modulatory effects of cocoa, and to establish whether these effects depend on the rat strain. To this end, 3-week-old Wistar and Brown Norway rats were fed, for 4 weeks, either a standard diet or the following three isoenergetic diets containing increasing proportions of cocoa flavonoids from different sources: one with $0.2 \%$ polyphenols (from conventional defatted cocoa), and two others with $0 \cdot 4$ and $0 \cdot 8 \%$ polyphenols (from non-fermented cocoa, very rich in polyphenols). Serum Ig concentrations, faecal IgA levels, microbiota composition and IgA-coating bacterial proportion were evaluated at the beginning and at the end of the study. After the nutritional intervention, the composition of lymphocytes in Peyer's patches and mesenteric lymph nodes was evaluated. In some respects, the Wistar strain was more sensitive to the impact of the cocoa diets than the Brown Norway strain. After 4 weeks of dietary intervention, similar modulatory effects of the diets containing $0 \cdot 2$ and $0.8 \%$ polyphenols on mucosal IgA levels and microbiota composition were found, although the $0 \cdot 2 \%$ diet, with a higher proportion of theobromine and fibre, had more impact, suggesting that polyphenols are not the only components involved in such effects.
\end{abstract}

Key words: Cocoa: Immune system: Intestinal immune system: Immunoglobulins: Microbiota

Cocoa powder is a product derived from the beans of the Theobroma cacao plant that is considered a rich source of fibre (26-40\%), proteins (15-20\%), carbohydrates (about 15\%) and lipids (10-24\%). It also provides minerals and vitamins ${ }^{(1)}$. It has become a subject of increasing interest due to its high content of flavonoids, compounds with antioxidant activity. Cocoa mainly contains the monomers (-)-epicatechin and catechin as well as various polymers derived from both of these compounds, known as procyanidins. Methylxanthines have also been identified in cocoa powder ${ }^{(2)}$.

To date, there have not been many studies addressing the dietary effects of polyphenols on gut-associated lymphoid tissue $\left(\right.$ GALT) ${ }^{(3)}$, and there have been even fewer studies about the effects of cocoa polyphenols on the functionality or composition of GALT in animals ${ }^{(4)}$. GALT constitutes the most extensive and complex part of the immune system in the body. Structurally, it is composed of diffused cells, considered to be the effector site of the immune response (intraepithelial and lamina propria lymphocytes), and organised lymphoid structures considered as the inductive site of the intestinal immune response (Peyer's patches (PP), isolated follicles and mesenteric lymph nodes (MLN)). Previous studies $^{(5)}$ have reported that the ratios of $\mathrm{T}: \mathrm{B}$ lymphocytes and Th:Tc cell subsets in PP and MLN are modified after a high intake of cocoa polyphenols in young rats.

IgA is the most abundant Ig isotype produced in the body, and it is estimated that about $80 \%$ of all IgA antibody-secreting cells reside in the gut mucosa ${ }^{(6)}$. This antibody constitutes the first line of non-inflammatory immune protection at mucosal surfaces by neutralising microbial pathogens and exotoxins and by interacting with innocuous dietary antigens and commensal microbes ${ }^{(7,8)}$. The capacity to modulate secretory IgA (sIgA) levels by different polyphenols has been described previously $^{(9,10)}$. Focusing on cocoa polyphenols, their effects on sIgA levels have been studied in different experimental designs using rats that have confirmed that polyphenols

Abbreviations: FBS, fetal bovine serum; FCM, flow cytometry; FSC, forward-scatter characteristics; GALT, gut-associated lymphoid tissue; mAb, monoclonal antibodies; MLN, mesenteric lymph nodes; NK, natural killer; PC0 2 , diet containing $0 \cdot 2 \%$ polyphenol from conventional cocoa; PC0 4 , diet containing $0 \cdot 4 \%$ polyphenol from non-fermented cocoa rich in polyphenols; PC0 8 , diet containing $0 \cdot 8 \%$ polyphenol from non-fermented cocoa rich in polyphenols.; PP, Peyer's patches; REF, reference; RPMI, Roswell Park Memorial Institute; sIgA, secretory IgA; SSC, side-scatter characteristics; TCR, T-cell receptor.

*Corresponding author: F. J. Pérez-Cano, fax +349340359 01, email franciscoperez@ub.edu 
reduce $\operatorname{sIgA}$ protein and $\operatorname{sIgA}$ gene expression, which leads to a different pattern of IgA-coating bacteria ${ }^{(5,11,12)}$.

The crosstalk between GALT and the microbiota has been shown to be critical for mucosal tissue homeostasis, maintenance of mucosal barrier function and protection against infectious and inflammatory diseases occurring at mucosal sites. The interaction is a complex and tightly regulated process that distinguishes invasive pathogens and innocuous antigens from food and commensal bacteria ${ }^{(13)}$. During the metabolism of food and xenobiotics such as polyphenols, the host and its gut microbiota co-produce a large number of small molecules, many of which play critical roles in this crosstalk $^{(13)}$. Some of these signals from commensal intestinal bacteria continuously support the maintenance of mucosal lymphoid tissue ${ }^{(14)}$.

It has also been also observed that polyphenol-rich dietary sources have an influence on the gut microbiota ${ }^{(15,16)}$. In relation to this, it has recently been demonstrated that high intakes of polyphenols from cocoa are able to produce changes in microbiota composition in animals ${ }^{(12)}$ and in humans ${ }^{(17)}$. These changes in the microbiota could be partly responsible for the changes observed in GALT. The aim of the present study was to ascertain whether cocoa flavonoid content is uniquely responsible for the modulatory effects of cocoa on the immune system and microbiota as reported in previous studies, and at the same time, to evaluate whether these changes are rat strain dependent. To achieve this objective, two rat strains with a different immune response pattern were fed diets containing the following three different proportions of polyphenols from different sources of cocoa: one diet contained a conventional cocoa powder and the other two diets contained flavonoid-enriched cocoa powders obtained from non-fermented, blanched and non-roasted cocoa beans.

\section{Materials and methods}

\section{Diets}

A conventional natural Forastero cocoa powder (Nutrexpa S.L.) containing $21.20 \mathrm{mg} / \mathrm{g}$ of polyphenols was used to elaborate a diet with $0 \cdot 2 \%$ polyphenol (PCO 2 ). A cocoa polyphenol-enriched extract obtained from non-fermented, blanched and non-roasted cocoa beans and containing $160.3 \mathrm{mg} / \mathrm{g}$ was used to elaborate a diet with $0.4 \%$ polyphenol (PC0.4). Moreover, a 0.8\% polyphenol diet (PC0.8) was elaborated using an ethanol extract from the same non-fermented, blanched and non-roasted cocoa beans and containing $510 \mathrm{mg} / \mathrm{g}$ of polyphenols. The polyphenol characterisation of the three cocoa powders is summarised in Table 1.

The three cocoa diets were elaborated from a standard AIN$93 \mathrm{M}$ formula by subtracting the amount of carbohydrates, proteins, lipids and insoluble fibre provided by each cocoa source. The resulting cocoa polyphenol-rich chow diets were isoenergetic and had the same proportion of macronutrients (carbohydrates, proteins and lipids) and insoluble fibre as the standard diet, and provided the corresponding quantity of particular compounds such as cocoa soluble and insoluble fibre and cocoa polyphenols (Table 1).
Table 1. Composition of nutrients and polyphenols provided by the three cocoa powders in the experimental diets $(\mathrm{g} / \mathrm{kg}$ diet)

\begin{tabular}{|c|c|c|c|c|}
\hline Components & $\begin{array}{c}\text { REF } \\
\text { (AIN-93M, } \\
\text { g/kg) }\end{array}$ & $\begin{array}{r}\text { PC0.2 } \\
(\mathrm{g} / \mathrm{kg})\end{array}$ & $\begin{array}{l}\mathrm{PC} 0.4 \\
(\mathrm{~g} / \mathrm{kg})\end{array}$ & $\begin{array}{l}\mathrm{PC} 0.8 \\
(\mathrm{~g} / \mathrm{kg})\end{array}$ \\
\hline Casein & $121 \cdot 8$ & $97 \cdot 1$ & $115 \cdot 4$ & 119.5 \\
\hline L-Cystine & 1.8 & 1.4 & 1.8 & $1 \cdot 8$ \\
\hline Maize starch & $419 \cdot 1$ & $421 \cdot 0$ & $415 \cdot 7$ & 417.5 \\
\hline Maltodextrin & $147 \cdot 3$ & $116 \cdot 8$ & $147 \cdot 3$ & $147 \cdot 3$ \\
\hline Sucrose & $117 \cdot 0$ & $108 \cdot 7$ & $117 \cdot 0$ & $117 \cdot 0$ \\
\hline Soyabean oil & $40 \cdot 0$ & $26 \cdot 2$ & 37.7 & 38.9 \\
\hline Cellulose & $50 \cdot 0$ & 24.5 & $42 \cdot 9$ & $46 \cdot 5$ \\
\hline Minerals & $27 \cdot 0$ & $27 \cdot 8$ & $27 \cdot 0$ & $27 \cdot 0$ \\
\hline Vitamins & $2 \cdot 0$ & $7 \cdot 2$ & $2 \cdot 0$ & $2 \cdot 0$ \\
\hline Choline bitartrate & 2.5 & $2 \cdot 0$ & 2.5 & 2.5 \\
\hline tert-Butylhydroquine & 0.008 & 0.006 & 0.008 & 0.008 \\
\hline Cocoa powder & - & 100 & 25 & $17 \cdot 4$ \\
\hline Protein & - & 22 & 4.61 & $2 \cdot 26$ \\
\hline Carbohydrate & - & 16 & 3.35 & 1.64 \\
\hline Lipid & - & 11 & $2 \cdot 30$ & $1 \cdot 12$ \\
\hline Fibre & - & 34 & $7 \cdot 12$ & 3.5 \\
\hline Insoluble & & $25 \cdot 5$ & $5 \cdot 34$ & $2 \cdot 63$ \\
\hline Soluble & & 8.5 & 1.78 & 0.88 \\
\hline Total polyphenols* & - & $2 \cdot 12$ & 4 & 8.88 \\
\hline Catechin & - & 0.073 & 0.080 & 0.097 \\
\hline Epicatechin & - & 0.204 & 0.500 & 1.379 \\
\hline Isoquercetin & - & 0.0053 & ND & ND \\
\hline Quercetin & - & 0.0029 & ND & ND \\
\hline Procyanidin B1 & - & ND & 0.045 & 0.254 \\
\hline Procyanidin B2 & - & 0.167 & 0.310 & 0.713 \\
\hline Total procyanidins & - & ND & $3 \cdot 200$ & 7.795 \\
\hline
\end{tabular}

REF, reference diet; PC0.2, diet containing $0.2 \%$ polyphenol from conventional cocoa; PC0.4, diet containing $0.4 \%$ polyphenol from non-fermented cocoa rich in polyphenols; PC0.8, diet containing $0.8 \%$ polyphenol from non-fermented cocoa rich in polyphenols; ND, not determined.

* Total polyphenol compounds were estimated by the Folin-Ciocalteu spectrophotometric method. The polyphenol profile was identified and quantified using a reversed-phase HPLC coupled to a diode array detector, and the procyanidin profile (from monomers to > decamers) by normal-phase HPLC coupled to MS with fluorescence detection.

\section{Animals}

Female Wistar and Brown Norway rats (3 weeks old) were obtained from Harlan and housed in cages under conditions of controlled temperature and humidity in a $12 \mathrm{~h}$ light $-12 \mathrm{~h}$ dark cycle. After an acclimatisation period of 1 week, the rats were randomly distributed into four dietary groups ( $n 6$ per group) for each strain. The reference (REF) groups were fed with a standard AIN-93M diet (Harlan), and the cocoa groups received the $\mathrm{PC} 0 \cdot 2, \mathrm{PC} 0.4$ or $\mathrm{PC} 0 \cdot 8$ diets. The diets lasted for 4 weeks.

The rats were given free access to water and chow ad libitum. Body weight and food intake were monitored throughout the experiment. The studies were performed according to the criteria outlined by the Guide for the Care and Use of Laboratory Animals. Experimental procedures were reviewed and approved by the Ethical Committee for Animal Experimentation of the University of Barcelona (reference no. 358/12).

\section{Sample collection and processing}

Blood samples were collected weekly from the beginning of the diet until the end of the study, and after centrifugation, serum was separated and kept at $-20^{\circ} \mathrm{C}$ until Ig quantification 
by ELISA. Faecal samples were collected at the same time points and were processed for Ig quantification and bacterial characterisation. For this purpose, faecal homogenates were obtained, as described previously ${ }^{(5,12)}$, and frozen at $-20^{\circ} \mathrm{C}$ until analysis.

After 4 weeks of dietary intervention, the rats were anaesthetised intramuscularly with ketamine $(90 \mathrm{mg} / \mathrm{kg}$; Merial Laboratories, S.A., Barcelona) and xylazine $(10 \mathrm{mg} / \mathrm{kg}$; Bayer). The small intestine and MLN were collected. The small intestine was divided into two halves and carefully rinsed with cold $0.9 \% \mathrm{NaCl}$ solution in order to remove faecal content. The distal half was opened lengthwise and visible PP were excised. The intestinal tissue without PP was used to obtain the gut wash for IgA quantification by ELISA, as established previously in our laboratory ${ }^{(5)}$, while the proximal half was rejected. MLN and PP were used to isolate lymphocytes and to perform their phenotypic characterisation.

\section{Lymphocyte isolation from Peyer's patches and mesenteric lymph nodes}

The excised PP were incubated in a shaker with Roswell Park Memorial Institute (RPMI) medium containing $1 \mathrm{~mm}$-dithiothreitol (Sigma-Aldrich) for $5 \mathrm{~min}$ at $37^{\circ} \mathrm{C}$. Thereafter, PP were washed with RPMI medium and passed through a $40 \mu \mathrm{m}$ mesh (BD Biosciences). The filtered cell suspension was resuspended in RPMI medium containing $10 \%$ fetal bovine serum (FBS; Sigma-Aldrich) and 0.05 mm-2- $\beta$-mercaptoethanol (Merck). Cell counting and viability were determined by double staining with acridine orange and ethidium bromide (Sigma-Aldrich), followed by fluorescence light microscopy analysis using a Zeiss microscope (Axioskip MC80; Zeiss).

MLN cell suspension was obtained by passing the tissue through a sterile $40 \mu \mathrm{m}$ mesh and incubating on ice to remove tissue debris by sedimentation for $10 \mathrm{~min}$. Then, the suspensions were centrifuged $\left(500 \mathrm{~g}, 5 \mathrm{~min}, 4^{\circ} \mathrm{C}\right)$, and the cells were resuspended in RPMI medium containing $10 \%$ FBS and $0.05 \mathrm{~mm}-2-\beta$-mercaptoethanol. Cell counting and viability were also determined.

\section{Lymphocyte immunofluorescence staining and flow cytometry analysis}

PP and MLN lymphocytes were stained with anti-rat monoclonal antibodies (mAb) followed by flow cytometry (FCM) analysis. Mouse anti-rat mAb conjugated to fluorescein isothiocyanate, phycoerythrin or peridinin chlorophyll protein used herein included the following: anti-CD4 (OX-35); anti-T-cell receptor (TCR)- $\alpha \beta$ (R73); anti-CD8 $\alpha$ (OX-8); anti-CD25 (OX-39); antiCD-161a (10/78); anti-CD45RA (OX-33) (BD Biosciences); anti-IgA (Thermo Fisher Scientific). Cells $\left(2 \times 10^{5}\right)$ were labelled with saturating concentrations of conjugated mAb in PBS (pH 7.2) containing $1 \%$ FBS and $0.09 \%$ sodium azide (Merck) for $30 \mathrm{~min}$ at $4^{\circ} \mathrm{C}$ in the dark. Negative control staining using isotype-matched $\mathrm{mAb}$ was included for each sample. After washing with PBS, the cells were fixed with $0.5 \% p$-formaldehyde (Merck) and stored at $4{ }^{\circ} \mathrm{C}$ in the dark. Analyses were performed using an FC 500 Series Flow Cytometer (Beckman Coulter, Inc.), and data were assessed by the FlowJo version 7.6.5 software (Tree Star, Inc.). Lymphocyte populations were defined as follows: Th (TCR $\left.\alpha \beta^{+} \mathrm{CD} 4^{+}\right)$cells; Tc $\left(\mathrm{TCR} \alpha \beta^{+} \mathrm{CD} 8^{+}\right)$cells; natural killer (NK) T $\left(\mathrm{TCR} \alpha \beta^{+} \mathrm{NK}^{+}\right)$cells; NK $\left(\mathrm{TCR} \alpha \beta^{-} \mathrm{NK}^{+}\right)$cells; $\mathrm{B}\left(\mathrm{CD} 45 \mathrm{RA}^{+} \mathrm{CD}^{-}\right)$cells. Results are expressed as percentages of positive cells in the lymphocyte population selected according to their forward-scatter characteristics (FSC) and side-scatter characteristics (SSC), as reported previously ${ }^{(5)}$.

\section{Ig quantification in serum, faeces and gut washes by ELISA}

The amounts of sIgA in gut washes and faeces, and serum IgA, IgG1, IgG2a, IgG2b concentrations were quantified by ELISA, as described previously ${ }^{(11)}$. In brief, ninety-six-well polystyrene plates (Nunc MaxiSorp) were coated with anti-rat IgA, IgG1, IgG2a or IgG2b ( $2 \mathrm{mg} / \mathrm{ml}$ in PBS; BD Biosciences) and incubated overnight. After blocking with PBS containing $1 \%$ bovine serum albumin $(1 \mathrm{~h})$, the plates were washed and appropriate diluted samples and standard dilutions were added ( $3 \mathrm{~h})$. After washing, biotin-conjugated anti-rat IgA, IgG1, IgG2a or IgG2b mAb were added $(1 \mathrm{mg} / \mathrm{ml}$; BD Biosciences) and after $2 \mathrm{~h}$, peroxidase-conjugated ExtrAvidin ( $4 \mathrm{mg} / \mathrm{ml}$; Sigma-Aldrich) was incubated for $30 \mathrm{~min}$. Finally, the enzyme-substrate reaction was developed and stopped by adding $3 \mathrm{M}-\mathrm{H}_{2} \mathrm{SO}_{4}$ (Merck). Absorbance was measured in a microplate photometer (LabSystems Multiskan) at $492 \mathrm{~nm}$. Data were interpolated using ASCENT version 2.6 software (Thermo Fisher Scientific) into the standard curves, and expressed as $\mu \mathrm{g} / \mathrm{ml}$ for $\operatorname{IgG} 1$, IgG2a and $\operatorname{IgG} 2 \mathrm{~b}$ and as $\mathrm{ng} / \mathrm{ml}$ for IgA results.

\section{Fluorescence in situ hybridisation of gut microbiota}

The bacterial groups present in the faeces were characterised using the fluorescence in situ hybridisation technique with group- or genus-specific fluorochrome-conjugated probes (Sigma-Aldrich), as established previously in our laboratory ${ }^{(12)}$ with some modifications. The specific probes and controls used in the present study, as well as the hybridisation conditions, are summarised in Table 2. The EUB338 probe labelled at the $5^{\prime}$-end with indocarbocyanine fluorochrome (Cy3) was used to label total bacteria, whereas the NONEUB338-indodicarbocyanine fluorochrome (Cy5) probe was applied as a negative control in order to eliminate background fluorescence. Briefly, fixed cell suspensions were incubated in the presence of each fluorescent probe $(50 \mathrm{ng} / \mu \mathrm{l})$ in preheated hybridisation buffer (10 mm-Tris-HCl, $\mathrm{pH} 8,0.9 \mathrm{M}-$ $\mathrm{NaCl}$ and $0 \cdot 1 \%$ SDS) in a $0.2 \mathrm{ml}$ tube in a thermocycler at appropriate temperature (Table 2 ) for $2 \mathrm{~h}$ in the dark. In the case of Lactobacillus, samples were washed with Tris-EDTA buffer and permeabilised with $0.25 \mathrm{mg} / \mathrm{ml}$ of lysozyme (Serva) for $1 \mathrm{~h}$ at room temperature before the hybridisation process with the same hybridisation buffer but adding 30\% formamide. Hybridised cell suspensions were then washed with hybridisation buffer with $0.001 \%$ SDS for $30 \mathrm{~min}$ at the same temperature as hybridisation was done in order to eliminate non-specific binding of the probes. The hybridised cells 
Table 2. Oligonucleotide probes and hybridisation conditions used in the analysis of intestinal bacteria by fluorescence in situ hybridisation-flow cytometry analysis

\begin{tabular}{|c|c|c|c|c|}
\hline Target bacterial groups & Probe & Sequence $\left(5^{\prime}-3^{\prime}\right)$ & Hybridisation conditions $\left({ }^{\circ} \mathrm{C}\right)$ & References \\
\hline \multirow[t]{3}{*}{ Domain bacteria } & EUB338-I & GCTGCCTCCCGTAGGAGT & 46 & \multirow[t]{3}{*}{ Daims et al. ${ }^{(36)}$} \\
\hline & EUB338-II & GCAGCCACCCGTAGGTGT & 46 & \\
\hline & EUB338-III & GCTGCCACCCGTAGGTGT & 46 & \\
\hline Bacteroides/Prevotella & Bac303 & CCAATGTGGGGGACCTT & 46 & Manz et al. ${ }^{(37)}$ \\
\hline Bifidobacterium & Bif164 & CATCCGGCATTACCACCC & 50 & Langendijk et al. ${ }^{(38)}$ \\
\hline Lactobacillus/Enterococcus & Lab158 & GGTATTAGCAYCTGTTTCCA & 50 (lysozyme) & Harmsen et al. ${ }^{(39)}$ \\
\hline Escherichia coli & Ec1531 & CACCGTAGTGCCTCGTCATCA & 37 & Poulsen et al. ${ }^{(40)}$ \\
\hline Clostridium histolyticum/C. Perfringens & CHis 150 & TTATGCGGTATTAATCTYCCTTT & 50 & Franks et al. ${ }^{(41)}$ \\
\hline Staphylococcus spp. & Staphy & TССTССАТАТСТСTGCGC & 46 & Trebesius et al. ${ }^{(42)}$ \\
\hline Streptococcus spp. & Strept & САСТСТССССТТСТGСАС & 46 & Trebesius et al. ${ }^{(42)}$ \\
\hline Negative control & NON-EUB & ACTCCTACGGGAGGCAGC & 46 & Wallner et al. ${ }^{(43)}$ \\
\hline
\end{tabular}

were collected by centrifugation $(12000 \mathrm{~g}, 5 \mathrm{~min})$, washed twice in PBS, resuspended for FCM analysis and kept in the dark at $4^{\circ} \mathrm{C}$ until analysis.

\section{Ig-coating bacterial staining}

Faecal homogenates were diluted in $1 \%(\mathrm{v} / \mathrm{v})$ FBS/PBS and centrifuged $\left(8000 \mathrm{~g}, 5 \mathrm{~min}, 4^{\circ} \mathrm{C}\right)$. The resulting pellet was resuspended in $1 \%(\mathrm{v} / \mathrm{v})$ FBS/PBS containing fluorescein isothiocyanate-anti-rat IgA antibodies (Abcam). Each mixture was incubated for $30 \mathrm{~min}$ in the dark, washed twice with PBS ( $8000 \mathrm{~g}, 5 \mathrm{~min}$ ) and resuspended in PBS until analysis. A nonstained mixture of each sample was used as the control. To label total bacteria, the samples were mixed with propidium iodide (Sigma-Aldrich) before FCM analysis.

\section{Flow cytometry bacterial analysis}

For bacterial characterisation and IgA-coated bacterial determination, FCM analysis was performed using a FACSAria SORP sorter (BD Biosciences), as described previously ${ }^{(12)}$. Bacterial morphology was selected according to their FSC/ SSC signal. Subsequently, bacteria hybridised with group- or genus-specific probes conjugated to $\mathrm{Cy} 5$ or $\mathrm{Cy} 3$ were detected by combining the FSC signal with fluorescence detectors. FCM parameters were adjusted for bacterial counts, and bacterial concentrations of the samples were adjusted to obtain particle counts of 100-1000 events/s, and the events were recorded for $60 \mathrm{~s}$ in list mode files. Commercial Flow-Check ${ }^{\mathrm{TM}}$ Fluorospheres (Beckman Coulter, Inc.) were used to determine total counts combined with the EUB338-Cy3 probe. The proportion of IgA-coating bacteria was expressed as positive fluorescein isothiocyanate counts within the propidium iodide-positive bacteria present in the above FSC/SSC morphology gate. Both analyses were performed using the FlowJo version 7.6.5 software (Tree Star, Inc.)

The results of microbiota composition are expressed as the percentage of each genus with respect to the total bacterial population hybridised with EUB probes in each sample. Similarly, the results of IgA-coating bacteria are expressed as the percentage of bacterial cells labelled with anti-rat IgA with respect to the total cell population labelled with propidium iodide.

\section{Statistical analysis}

Statistical analysis was performed using the software package SPSS 18.0 (SPSS, Inc.). Levene's and Kolmogorov-Smirnov tests were applied to assess variance equality and normal distribution, respectively. Conventional one-way ANOVA was performed when normal distribution and equality of variance existed. The Bonferroni test was applied when specific cocoa intake had a significant effect on the dependent variable. Non-parametric Mann-Whitney $U$ and Wilcoxon tests were used in order to assess significance for independent and related samples, respectively. Significant differences were established at $P<0.05$.

\section{Results}

\section{Effects of cocoa polyphenols on body weight and chow intake}

During the study, although the body weight of Brown Norway rats was lower than that of Wistar rats, its slope was similar among the dietary groups in every rat strain with the exception of those that received the diet with lower polyphenol content (PC0.2 group). In Wistar rats, the PC0.2 diet produced a statistically significant lower increase than that in the REF groups during the study $(P<0.05$; Fig. $1(\mathrm{a}))$, whereas in Brown Norway rats, the significance appeared only at the end of the study $(P<0.05$; Fig. 1(b)). This effect was not related to lower chow intake since it was similar throughout the study among all the experimental groups in both rat strains (data not shown).

\section{Effects of cocoa polyphenols on serum IgG1, IgG2a and IgG2b concentrations}

Serum concentrations of IgG1, IgG2a and IgG2b from all the experimental groups throughout the study in both strains are summarised in Fig. 2. Wistar rats showed an age-increasing pattern in the second half of the study in the three IgG isotype concentrations (Fig. 2(a)-(c)). With regard to the nutritional intervention, the diets containing cocoa polyphenols partially reduced this increase in comparison with the REF diet, being statistically significant $(P<0.05)$ for IgG1 at day 21 (Fig. 2(a)) and for IgG2b at day 14 in the PCO.2 group and at the end 

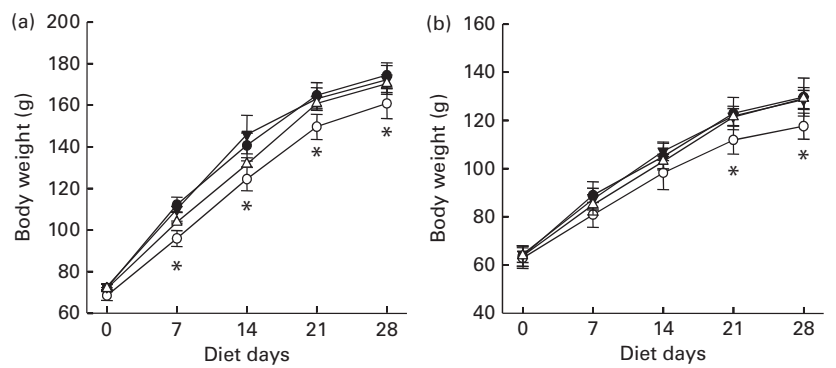

Fig. 1. Effects of cocoa polyphenol-enriched diets on body weight in (a) Wistar rats and (b) Brown Norway rats. Values are means $(n 6)$, with their standard errors represented by vertical bars. *Mean value was significantly different from that of the reference group $(--; P<0.05)$ on the same day of the study. - - - Diet containing $0.2 \%$ polyphenol from conventional cocoa; $\boldsymbol{\nabla}$, diet containing $0.4 \%$ polyphenol from non-fermented cocoa rich in polyphenols; $\triangle$, diet containing $0.8 \%$ polyphenol from non-fermented cocoa rich in polyphenols.

of the study after the intake of the three diets (Fig. 2(c)). No significant effects were observed for IgG2a.

Brown Norway rats showed higher IgG concentrations than Wistar rats, and at the end of the study, their values were 9-fold the IgG1 concentration (Fig. 2(d)), 3-fold the IgG2a concentration (Fig. 2(e)) and 2-fold the IgG2b concentration (Fig. 2(f)) compared with those found in Wistar rats. No significant effects were observed after the nutritional interventions with any of the cocoa polyphenol-enriched diets in this rat strain with the exception of a punctual increase in IgG1 concentration by the PCO 2 diet.

\section{Effects of cocoa polyphenols on serum, faecal and gut wash IgA concentration}

Serum obtained during the period of the study was also used to quantify IgA. There were no effects on IgA concentration in Wistar rats after any nutritional interventions (Fig. 3(a)). On the contrary, Brown Norway rats fed with cocoa polyphenols decreased serum IgA concentration after 3 weeks of diets, the concentration being significantly lower than the REF groups $(P<0.05)$, though at the end of the study, the levels were similar among all the dietary groups (Fig. 3(b)).

Intestinal IgA concentration was also quantified in the faeces and gut washes. Faecal IgA concentration quickly increased over the study period in REF Wistar rats. This agedependent increase was partially but significantly avoided by all cocoa polyphenol-enriched diets $14 \mathrm{~d}$ after the nutritional intervention $(P<0 \cdot 05)$. Nevertheless, the inhibitory effect on faecal IgA remained significant only in rats that were fed with the PCO. 2 and PCO. 8 diets at the end of the study (Fig. 3(c)). The age-dependent increase in faecal IgA concentration observed in the Wistar strain was also observed in REF Brown Norway rats for the first 3 weeks of the study. The three polyphenol-enriched diets partially avoided such an increase $(P<0.05)$. After 1 week, faecal IgA concentration was similar among the dietary groups (Fig. 3(d)).

The results obtained for gut wash IgA at the end of the study were similar in both strains (Fig. 3(e) and (f)). All the three experimental diets reduced their concentrations, but they were only statistically significant in rats fed with the PCO 2 and PC0.8 diets $(P<0.05)$, which showed up to four to five times lower IgA concentrations in gut washes.

\section{Effects of cocoa polyphenols on lymphocyte composition in mesenteric lymph nodes and Peyer's patches}

The percentages of B, Th, Tc, NK and NKT cells in MLN and PP for both strains and each cocoa polyphenol-enriched diet are summarised in Fig. 4.

Although no significant effects were observed in any of the studied MLN cell populations from Wistar and Brown Norway rats after cocoa polyphenol-enriched diets, the PC0.8 diet tended to reduce Th proportion and at the same time tended to increase B cells (Fig. 4(a) and (b)).

With regard to the PP lymphocyte population, all three polyphenol-enriched diets have a tendency to up-regulate the NK proportion in both studied strains, but only the PCO 2 diet significantly increased its proportion in Wistar rats $(P<0 \cdot 05$; Fig. $4(\mathrm{c})$ and $(\mathrm{d})$ ). Moreover, all diets tended to reduce the proportion of both Th and Tc cells with an increase in the proportion of $\mathrm{B}$ cells in Wistar rats. On the contrary, in Brown Norway rats, a tendency to increase the proportion of B cells while decreasing that of Th cells was only observed for the PC0.4 diet (Fig. 4(d)).

\section{Effects of cocoa polyphenols on gut microbiota composition}

As the Wistar strain showed a higher impact of the cocoa diets than the Brown Norway strain on faecal IgA concentration at the end of the study (Fig. 3(c) and (d)), the following determinations were only performed in Wistar rats.

The composition of faecal microbiota in Wistar rats was characterised by the FCM-fluorescence in situ hybridisation technique before the start of the diet and at the end of the study in all dietary groups (Table 3). All groups showed a similar microbiota composition pattern at baseline.

The proportion of Bacteroides, Bifidobacterium, Streptococcus and Staphylococcus increased by age in the REF groups $(P<0.05)$. With regard to the nutritional intervention, all the three cocoa polyphenol-enriched diets led to significant differences in microbiota composition. After 4 weeks of nutritional intervention, the rats fed the PCO 2 diet showed a significant decrease in the total counts of bacteria compared with their baseline values $(P<0.05)$ and with the REF diet $(P<0.05)$, and a lower proportion of Lactobacillus with respect to their baseline values $(P<0.05)$. This effect on Lactobacillus was also observed in the PC0.4 and PC0.8 groups. The age-dependent increase in Staphylococcus genera was significantly lower after the intake of the PCO. 2 diet than after the intake of the REF diet $(P<0.05)$. In contrast, the increase in the proportion of Streptococcus by age was inhibited by the PC0 2 and PCO 8 diets $(P<0 \cdot 05)$.

\section{Effects of cocoa polyphenols on IgA-coating bacteria}

The percentage of IgA-coating bacteria was determined by FCM according to their FSC/SSC signal using faecal samples collected 

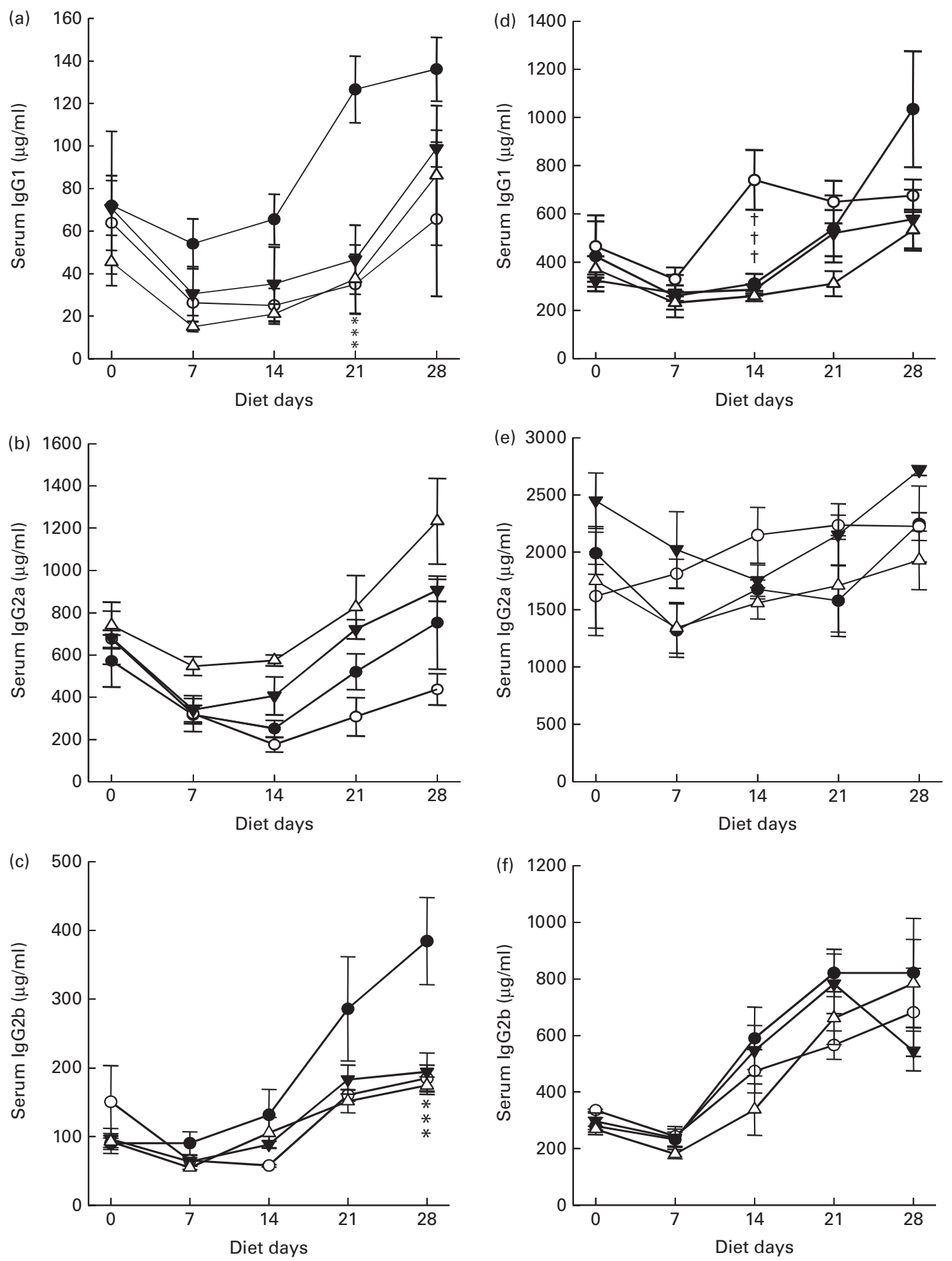

Fig. 2. Effects of cocoa polyphenol-enriched diets on serum IgG1 (a, d), IgG2a (b, e) and IgG2b isotypes (c, f) in (a-c) Wistar rats and (d-f) Brown Norway rats. Values are means $(n 6)$, with their standard errors represented by vertical bars. * ${ }^{*}$ ean value was significantly different from that of the reference group $(-\bullet$; $P<0.05$ ) on the same day of the study. †Mean value was significantly different from that of the group consuming the diet containing $0.2 \%$ polyphenol from conventional cocoa $(-0-; P<0.05)$ on the same day of the study. $\rightarrow$, Diet containing $0.4 \%$ polyphenol from non-fermented cocoa rich in polyphenols; $\triangle$, diet containing $0.8 \%$ polyphenol from non-fermented cocoa rich in polyphenols.

before the start of the diet and at the end of the study (Fig. 5(a)). At the beginning of the study, all groups showed similar IgAcoating patterns (Fig. 5(b)). This proportion decreased by age up to $10 \%$ in the REF groups at day 28 . The groups that received the PC0.2 and PC0.8 diets also had a decreased percentage of IgA-coating bacteria with respect to those found at baseline $(P<0.05)$, with the reduction by the PC0.2 diet being stronger than that by the REF diet $(P<0.05 ;$ Fig. $5(\mathrm{c}))$. 

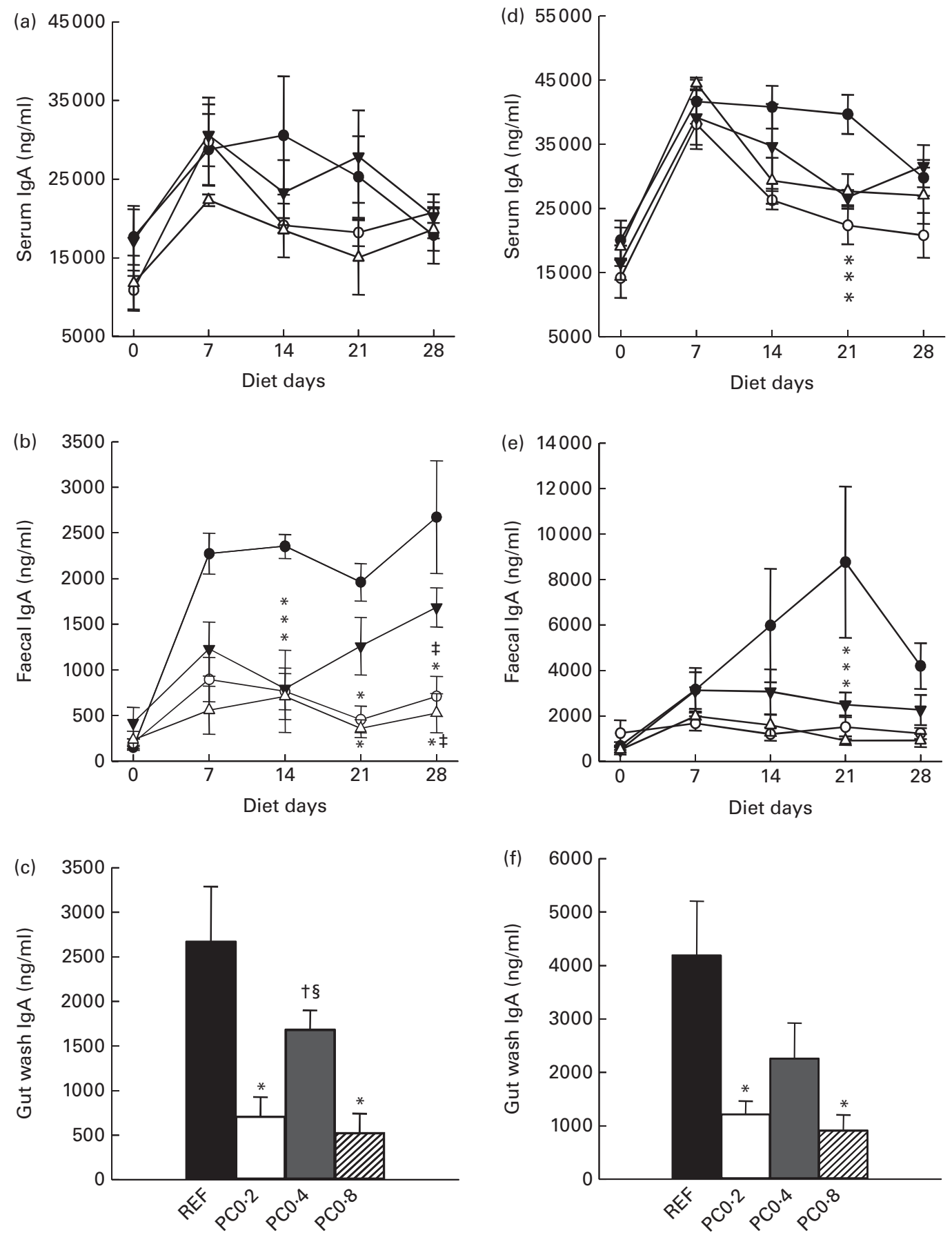

Fig. 3. Effects of cocoa polyphenol-enriched diets on systemic and intestinal IgA concentrations. Systemic IgA concentration was measured in serum samples from (a) Wistar rats and (d) Brown Norway rats. Intestinal IgA concentration was quantified in faecal samples (b, e) and in small-intestinal washes (c, f) from Wistar rats $(b, c)$ and Brown Norway rats $(e, f)$ at day 28 . Values are means $(n 6)$, with their standard errors represented by vertical bars. ${ }^{\star}$ Mean value was significantly different from that of the reference (REF) groups $(-; P<0.05)$ on the same day of the study. †Mean value was significantly different from that of the group consuming the diet containing $0.2 \%$ polyphenol from conventional cocoa (PC0.2,-0-; $P<0.05)$ on the same day of the study. $\ddagger$ Mean value was significantly different from that of the group consuming the diet containing $0.4 \%$ polyphenol from non-fermented cocoa rich in polyphenols (PC0.4, -7 ; $P<0.05$ ) on the same day of the study. §Mean value was significantly different from that of the group consuming the diet containing $0.8 \%$ polyphenol from non-fermented cocoa rich in polyphenols $(\mathrm{PCO} \cdot 8,-\rightarrow P<0.05)$ on the same day of the study.

\section{Discussion}

In previous studies, cocoa-enriched diets have demonstrated their immunomodulatory role in the systemic and intestinal compartments of rats ${ }^{(4)}$. It is believed that this effect is mainly due to the polyphenols present in cocoa. The present study focused on investigating whether these effects are attributed exclusively to them or should be attributed to other compounds present in cocoa, and, in addition, on evaluating 

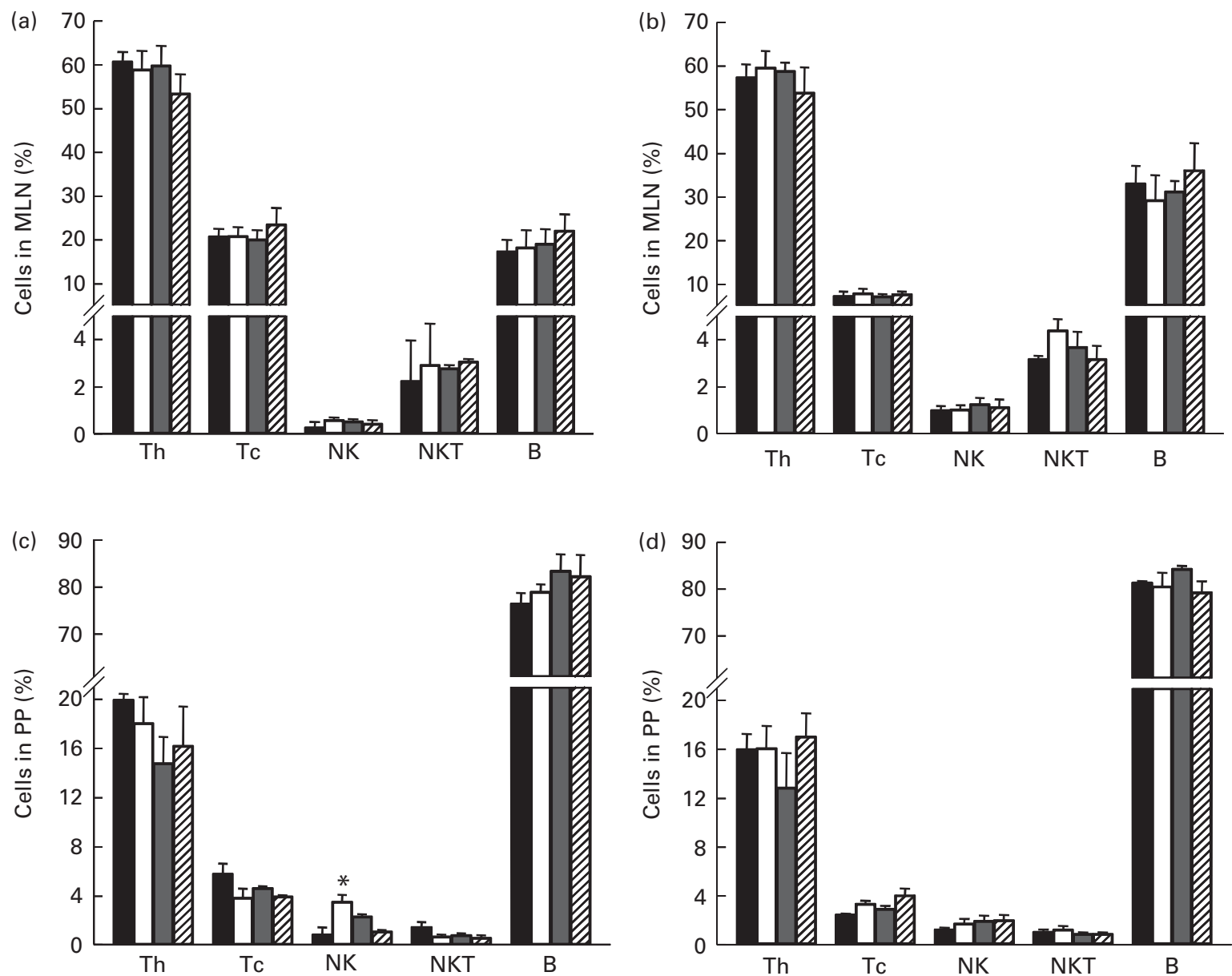

Fig. 4. Effects of cocoa polyphenol-enriched diets on (a, b) mesenteric lymph nodes (MLN) and (c, d) Peyer's patches (PP) from (a, c) Wistar rats and (b, d) Brown Norway rats. Values are means $(n 6)$, with their standard errors represented by vertical bars. *Mean value was significantly different from that of the reference group $(\square ; P<0.05)$. NK, natural killer. $\square$, Diet containing $0.2 \%$ polyphenol from conventional cocoa; $\square$, diet containing $0.4 \%$ polyphenol from non-fermented cocoa rich in polyphenols; $\mathbb{Z}$, diet containing $0.8 \%$ polyphenol from non-fermented cocoa rich in polyphenols.

whether these changes are rat strain dependent. To achieve this, two different rat strains were fed three cocoa diets formulated with three distinct cocoa raw materials differing in the proportion of polyphenols and other cocoa compounds. From the results, it emerges that Wistar rats are, in some aspects, more sensitive than Brown Norway rats to the effects of a cocoa diet on the immune system. In addition, the influence of cocoa on intestinal IgA and microbiota depends on polyphenols; however, as these effects are not dose dependent, other compounds must be involved in this influence.

Previous reports concerning the effect of cocoa on the immune system in vivo were developed using 2-10\% cocoa-enriched diets formulated with conventional cocoa containing, among other compounds, $20-30 \mathrm{mg} / \mathrm{g}$ of polyphenols ${ }^{(5,12,18)}$. The present study includes the effect of two cocoa powders very rich in polyphenols (about 160 and $500 \mathrm{mg} / \mathrm{g}$, respectively), because they were obtained without fermentation, allowing them to keep the polyphenols contained in cocoa seeds ${ }^{(19)}$. In the elaboration of the PC0.4 and PC0.8 diets, small amounts of cocoa powders were used to double or to quadruple the polyphenol content of the conventional $10 \%$ cocoa diet (PC0 2 ). The amount of conventional cocoa powder used to elaborate the $\mathrm{PCO} 2$ diet provided about $34 \mathrm{mg} / \mathrm{g}$ of fibre, including both soluble and insoluble fibre, and about $2 \mathrm{mg} / \mathrm{g}$ of theobromine. In summary, in the present study, three cocoa diets with increasing proportions of polyphenols $(0 \cdot 2-0 \cdot 8 \%)$ and decreasing content of other cocoa compounds, such as fibre and theobromine, were designed to dissect the effects of cocoa on the immune system.

First, the influence of cocoa polyphenol intake on the increase in body weight was evaluated during the study period. Although during this period, there was no difference regarding food intake among the experimental groups, those fed with the PCO. 2 diet in both rat strains had a lower increase in body weight, a result that is in line with previous studies carried out using conventional cocoa ${ }^{(12,18,20,21)}$. However, it is worth noting that diets containing non-fermented cocoa with a higher amount of polyphenols did not produce such an effect, suggesting, therefore, that the influence of cocoa on body weight does not depend on cocoa polyphenol content. In this sense, we can suggest that methylxanthines found in higher concentrations in the $\mathrm{PCO} \cdot 2$ diet than in the PC0.4 and PCO.8 diets could play an important role ${ }^{(22)}$. 
Table 3. Bacterial percentage of each genus with respect to total bacteria determined by fluorescence in situ hybridisation-flow cytometry analysis in the faeces

(Mean values with their standard errors)

\begin{tabular}{|c|c|c|c|c|c|c|c|c|c|c|c|c|c|c|c|c|}
\hline \multirow{3}{*}{$\begin{array}{l}\text { Group... } \\
\text { Genus }\end{array}$} & \multicolumn{8}{|c|}{ Before intervention } & \multicolumn{8}{|c|}{ After intervention } \\
\hline & \multicolumn{2}{|c|}{ REF } & \multicolumn{2}{|c|}{$\mathrm{PC} 0 \cdot 2$} & \multicolumn{2}{|c|}{$\mathrm{PC} 0.4$} & \multicolumn{2}{|c|}{$\mathrm{PC} 0.8$} & \multicolumn{2}{|c|}{ REF } & \multicolumn{2}{|c|}{$\mathrm{PC} 0 \cdot 2$} & \multicolumn{2}{|c|}{$\mathrm{PC} 0.4$} & \multicolumn{2}{|c|}{$\mathrm{PC} 0.8$} \\
\hline & Mean & SEM & Mean & SEM & Mean & SEM & Mean & SEM & Mean & SEM & Mean & SEM & Mean & SEM & Mean & SEM \\
\hline Total bacteria & $80 \cdot 24$ & $2 \cdot 67$ & $85 \cdot 60$ & $5 \cdot 25$ & $81 \cdot 17$ & 4.93 & 80.04 & $7 \cdot 10$ & $81 \cdot 19$ & $5 \cdot 19$ & $62 \cdot 30^{*} \dagger$ & 3.58 & $78 \cdot 29$ & $5 \cdot 33$ & $84 \cdot 26 \ddagger$ & $2 \cdot 03$ \\
\hline Bacteroides & $16 \cdot 82$ & 1.66 & $18 \cdot 87$ & 3.50 & $13 \cdot 87$ & 3.41 & 11.43 & 1.44 & $32 \cdot 81^{*}$ & 4.53 & $22 \cdot 24$ & 0.23 & 26.94 & 4.57 & 23.55 & $5 \cdot 26$ \\
\hline Bifidobacterium & 1.84 & 0.45 & 1.86 & 0.76 & 1.99 & 0.22 & 3.85 & 0.56 & $8 \cdot 64^{*}$ & 1.87 & 4.79 & 1.45 & 3.85 & $1 \cdot 18$ & 3.27 & 0.09 \\
\hline Lactobacillus & 11.00 & 3.33 & $18 \cdot 50$ & $1 \cdot 32$ & $19 \cdot 25$ & 0.75 & $11 \cdot 25$ & 0.75 & $5 \cdot 75$ & $1 \cdot 83$ & $4 \cdot 00^{\star}$ & $2 \cdot 02$ & $2 \cdot 83^{*}$ & 0.83 & $4 \cdot 83^{\star}$ & 0.60 \\
\hline Escherichia coli & 3.01 & 0.04 & 3.40 & 0.31 & $3 \cdot 79$ & 0.45 & $5 \cdot 38$ & 1.73 & 3.73 & 0.97 & 1.70 & 0.84 & 1.79 & 0.13 & 1.33 & 0.36 \\
\hline $\begin{array}{l}\text { Clostridium histolyticum/ } \\
\text { C. perfringens }\end{array}$ & $7 \cdot 00$ & 1.74 & $6 \cdot 50$ & 0.76 & 3.50 & 1.00 & 4.00 & 0.50 & 14.67 & 3.17 & $6 \cdot 17$ & 0.83 & $15 \cdot 00^{\star} \S$ & $2 \cdot 65$ & $4 \cdot 17$ & 1.09 \\
\hline Streptococcus & $25 \cdot 16$ & $5 \cdot 20$ & 34.42 & $5 \cdot 77$ & $27 \cdot 35$ & 5.06 & 33.36 & 5.49 & $57 \cdot 05^{\star}$ & $7 \cdot 26$ & 23.95† & $5 \cdot 73$ & 34.57 & $5 \cdot 14$ & $25 \cdot 27 \dagger$ & $7 \cdot 30$ \\
\hline Staphylococcus & 3.96 & $1 \cdot 16$ & 5.58 & 0.90 & $11 \cdot 81$ & 4.94 & 14.01 & 4.52 & $54 \cdot 60^{*}$ & 3.65 & $25 \cdot 32^{*} \dagger$ & 3.55 & $44.63^{\star} \ddagger$ & 1.50 & $43 \cdot 60^{*} \ddagger$ & 4.41 \\
\hline
\end{tabular}

REF, reference diet; PC0.2, diet containing $0.2 \%$ polyphenol from conventional cocoa; PC0.4, diet containing $0.4 \%$ polyphenol from non-fermented cocoa rich in polyphenols; $\mathrm{PC} 0.8$, diet containing $0.8 \%$ polyphenol from non-fermented cocoa rich in polyphenols.

${ }^{*}$ Mean value was significantly different from that before the intervention $(P<0.05)$.

$\dagger$ Mean value was significantly different from that of the REF group at the end of the study $(P<0.05)$.

$\ddagger$ Mean value was significantly different from that of the PC0.2 group at the end of the study $(P<0.05)$.

$\S$ Mean value was significantly different from that of the PC0.8 group at the end of the study $(P<0.05)$.

Further studies must delve into the cocoa compounds and the mechanisms involved in this effect.

One of the immune aspects considered in the present study is the influence of cocoa polyphenols on serum Ig concentrations. It has been reported that food polyphenols have the ability to modulate systemic Ig production ${ }^{(23,24)}$. In the present study, we observed that the three cocoa diets attenuated the age-increasing pattern observed for serum IgG1 and IgG2b concentrations in Wistar rats but not in Brown Norway rats. This effect did not depend on the polyphenol dose alone, thus suggesting that other compounds in the formulation of cocoa must have also contributed and even potentiated the effect when lower amounts of polyphenols are present. In contrast, serum IgG concentrations in Wistar rats are much lower than those in Brown Norway rats and, perhaps as a consequence, the first strain is more sensitive to cocoa intake than the latter one. The attenuation of serum $\operatorname{IgG}$ concentration produced by cocoa is in line with previous studies also carried out in Wistar rats ${ }^{(11)}$.

With regard to intestinal IgA, the present results from a conventional cocoa diet $(\mathrm{PCO} \cdot 2)$ confirmed the down-modulatory effect on intestinal (faecal and gut wash) IgA content shown in previous studies, including several proportions of cocoa in diets (2-10\%), different ages at the beginning of the dietary intervention (3-6 weeks old) and different lengths of the diet $(3-9 \text { weeks })^{(5,12,25)}$. The attenuating effect on intestinal IgA produced by the PCO 2 diet was also produced by nonfermented cocoa when used at $0.8 \%$ concentration in both Wistar and Brown Norway rats, in a similar way to what was observed in older Lewis rats fed with similar PCO.2 and PC0.8 diets for 2 weeks ${ }^{(21)}$. However, when considering IgAcoating bacteria, its percentage decreased after the intake of the PC $0 \cdot 2$ diet but not after the PC $0 \cdot 8$ diet, thereby partially reflecting the attenuating effect on intestinal IgA. The lowering effect of a conventional cocoa polyphenol diet (PC0.2) on bacteria coated with IgA observed herein is in line with previous studies conducted with a longer intervention in
Wistar rats ${ }^{(12)}$ and also in older Lewis rats ${ }^{(21)}$. Overall, these results suggest that polyphenols can be responsible for the decrease in intestinal IgA concentrations, but some other compounds present in conventional cocoa must have also contributed or potentiated the effect of polyphenols.

The study of lymphocyte composition in PP and MLN can shed light on the effect of cocoa on intestinal IgA. Some studies have reported the ability of polyphenols to modulate the proportion of some mucosal lymphocyte populations ${ }^{(26)}$, and also previous results have shown the effect of conventional cocoa on these compartments ${ }^{(5)}$. However, in the present study, the diets containing conventional cocoa did not produce profound changes with the exception of the increase of the NK population in PP lymphocytes. Similarly, diets containing a higher amount of polyphenols did not produce any effect on lymphocyte composition in MLN and PP. Since no significant changes were observed in the percentage of Th and B cells, the proportion of these cells in MLN or PP cannot explain the attenuating effect of cocoa polyphenols on intestinal IgA concentration, and this fact suggests that intracellular mechanisms, released immune mediators or the transcytosis of IgA across the intestinal wall could be responsible for the effect on intestinal IgA concentration ${ }^{(11)}$. In contrast, the present results support the up-modulatory effect of conventional cocoa on the proportion of NK cells in the PP of Wistar rats, and agree with the enhancing effect of cocoa on innate immunity, as described previously ${ }^{(5)}$.

With regard to the gut microbiota, a decrease in total bacteria counts and some composition changes were produced by 4 weeks of cocoa diets, this being more pronounced again with the lowest concentration of polyphenols provided by the conventional cocoa. In general, cocoa diets inhibited the growth of Staphylococcus, Streptococcus and Clostridium bistolyticum/C. perfringens (belonging to the Firmicutes phylum) produced by age. The inhibitory effect on the growth of Staphylococcus and Streptococcus genera induced by the PC0.2 diet is partially in line with previous findings in young 
(a)

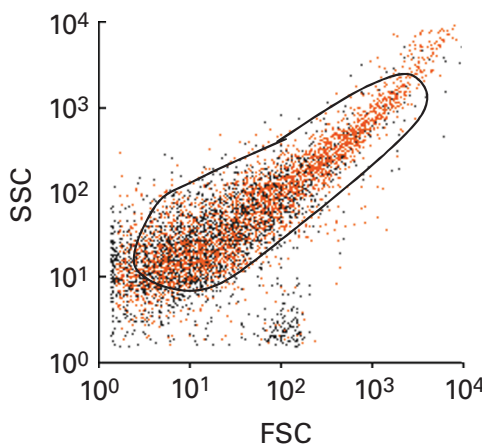

(b)

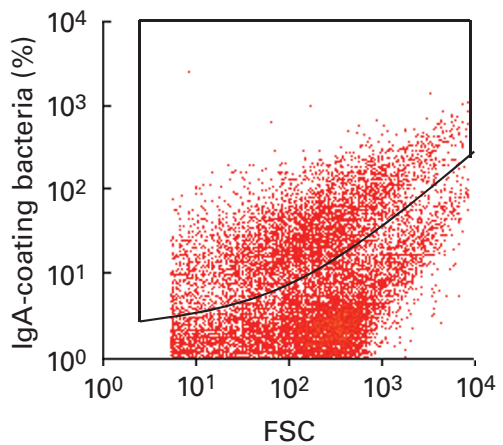

(c)

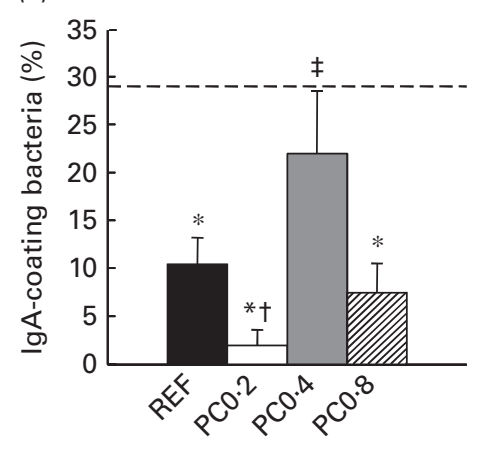

Fig. 5. Representative biparametric cytogram showing the (a) initial acquisition gate to select the bacterial population according to its forward-scatter characteristic (FSC)/side-scatter characteristic (SSC) signals. (b) Cytogram representing the mean of the percentage of IgA-coating bacteria, with their standard errors at baseline for all groups at day 0 (29.1 (SEM 3.3)). (c) IgA-coating bacteria at the end of the $28 \mathrm{~d}$ of nutritional intervention in all the groups. -- -, Basal value. Values are means $(n 6)$, with their standard errors represented by vertical bars. *Mean value was significantly different from that before the intervention $(P<0.05)$. $†$ Mean value was significantly different from that of the reference ( $\square$; REF) groups $(P<0.05)$ on the same day of the study. $\neq$ Mean value was significantly different from that of the group consuming the diet containing $0.2 \%$ polyphenol from conventional cocoa ( $\square$; PC0.2, $P<0.05$ ) on the same day of the study. $\square$, PC0.4, diet containing $0.4 \%$ polyphenol from non-fermented cocoa rich in polyphenols; $\varangle$, PC0.8, diet containing $0.8 \%$ polyphenol from non-fermented cocoa rich in polyphenols. (A colour version of this figure can be found online at http://www.journals.cambridge.org/bjn)

Wistar rats after 6 weeks of nutritional intervention ${ }^{(12)}$. In this context, polyphenols have been associated with an inhibitory effect on the growth of $C$. histolyticum/C. perfringens genera in in vitro, preclinical and clinical studies ${ }^{(12,17,27-30)}$. In contrast, the rats fed with the three cocoa polyphenol-enriched diets showed a decrease in the proportion of Bifidobacterium, which is not in line with other authors who attributed a bifidogenic effect to the polyphenols ${ }^{(17,29,31,32)}$. These differential results could be attributed to several factors such as the source of the polyphenols (pure flavonoids or polyphenol-rich dietary sources), dose, methodology (in vitro, preclinical or clinical studies) and differential composition and distribution ecosystems (rats $v$. human gut). In addition, it has been reported that polyphenols have diverse selective prebiotic effects and antimicrobial activities against certain genera of the gut microbiota $^{(33)}$, Gram-negative bacteria being more resistant than Gram-positive bacteria ${ }^{(34)}$, and excessive amounts of polyphenols may inhibit the growth of beneficial colonic microbiota ${ }^{(35)}$. In any case, from these data and unpublished results from our ongoing research, it could be suggested that cocoa polyphenols influence microbiota composition, but some other compounds, such as fibre and/or methylxanthines, must enhance this effect.

In summary, some immunomodulatory effects of diets containing high cocoa depend on the rat strain and could be partially attributed to their polyphenol content. Other cocoa compounds could also be key factors in this effect. Further studies evaluating dissected components of cocoa, such as fibre and theobromine, should be carried out in order to evaluate whether they are able to reproduce, at least partially, the previously and herein described cocoa effects on the intestinal immune system.

\section{Acknowledgements}

The authors thank Patricia Nevot for her technical assistance, and the Nutrexpa Group for providing the conventional cocoa powder.

The present study was supported by grants from the Spanish Ministries of Science and Innovation (grant no.
AGL2008-02790), and Economy and Competitivity (grant no. AGL2011-24279). M. M.-C. holds a fellowship from the Generalitat de Catalunya (grant no. 2013FI_B1 00140). None of the funders had a role in the design or analysis of the study or in the writing of this article.

The authors' contributions were as follows: M. M.-C., M. A.-G., À. F., M. C. and F. J. P.-C. conceived and designed the research; M. M.-C., S. T. and M. A.-G. carried out the experiments; M. M.-C., M. A.-G. and F. J. P.-C. carried out the data analysis; M. M.-C., M. C. and F. J. P.-C. were involved in the interpretation of the data; M. M.-C. contributed to the drafting of the manuscript; M. M.-C., M. A.-G., À. F., M. C. and F. J. P.-C. contributed to the critical revision of the manuscript; F. J. P.-C. had primary responsibility for the final content. All authors read and approved the final version of the manuscript.

None of the authors has any conflict of interest to declare.

\section{References}

1. Katz DL, Doughty K \& Ali A (2011) Cocoa and chocolate in human health and disease. Antioxid Redox Signal 15 2779-2811.

2. Tomás-Berberán FA, Cienfuegos-Jovellanos E, Marín A, et al (2007) A new process to develop a cocoa powder with higher flavonoid monomer content and enhanced bioavailability in healthy humans. J Agric Food Chem 55, 3926-3935.

3. Sehm J, Lindermayer H, Dummer C, et al. (2007) The influence of polyphenol rich apple pomare or red-wine pomace diet on the gut morphology in weaning piglets. J Anim Physiol Anim Nutr (Berl) 91, 289-296.

4. Pérez-Cano FJ, Massot-Cladera M, Franch A, et al. (2013) The effects of cocoa on the immune system. Front Pharmacol 4, 1-12.

5. Ramiro-Puig E, Pérez-Cano FJ, Ramos-Romero S, et al. (2008) Intestinal immune system of young rats influenced by cocoa-enriched diet. J Nutr Biochem 19, 555-565.

6. Mora JR \& von Andrian UH (2008) Differentiation and homing of IgA-secreting cells. Mucosal Immunol 1, 96-109.

7. Corthésy B (2007) Roundtrip ticket for secretory IgA: role in mucosal homeostasis? J Immunol 178, 27-32. 
8. MacPherson AJ, McCoy KD, Johansen FE, et al. (2008) The immune geography of IgA induction and function. Mucosal Immunol 1, 11-22.

9. Chen CM, Li SC, Lin YL, et al. (2005) Consumption of purple sweet potato leaves modulates human immune response: T-lymphocyte functions, lytic activity of natural killer cell and antibody production. World J Gastroenterol 11, $5777-5781$.

10. Okazaki Y, Han Y, Kayahata M, et al. (2010) Consumption of curcumin elevates fecal immunoglobulin A, an index of intestinal immune function, in rats fed a high-fat diet. $J$ Nutr Sci Vitaminol (Tokyo) 56, 68-71.

11. Pérez-Berezo T, Franch A, Ramos-Romero S, et al. (2011) Cocoa-enriched diets modulate intestinal and systemic humoral immune response in young adult rats. Mol Nutr Food Res 55, S56-S66.

12. Massot-Cladera M, Pérez-Berezo T, Franch A, et al. (2012) Cocoa modulatory effect on rat faecal microbiota and colonic crosstalk. Arch Biochem Biophys 527, 105-112.

13. Abreu MT (2010) Toll-like receptor signaling in the intestinal epithelium: how bacterial recognition shapes intestinal function. Nat Rev Immunol 10, 131-144.

14. Hill DA \& Artis D (2010) Intestinal bacteria and the regulation of immune cell homeostasis. Annu Rev Immunol $\mathbf{2 8}$, 623-667.

15. Etxeberria U, Fernández-Quintela A, Milagro FI, et al. (2013) Impact of polyphenols and polyphenol-rich dietary sources on gut microbiota composition. J Agric Food Chem 61, 9517-9533.

16. Van Duynhoven J, Vaughan EE, van Dorsten F, et al. (2013) Interactions of black tea polyphenols with human gut microbiota: implications for gut and cardiovascular health. Am J Clin Nutr 98, 1631S-1641S.

17. Tzounis X, Rodríguez-Mateos A, Vulevic J, et al. (2011) Prebiotic evaluation of cocoa-derived flavanols in healthy humans by using a randomized, controlled, double-blind, crossover intervention study. Am J Clin Nutr 93, 62-72.

18. Pérez-Berezo T, Ramiro-Puig E, Pérez-Cano FJ, et al. (2009) Influence of a cocoa-enriched diet on specific immune response in ovalbumin-sensitized rats. Mol Nutr Food Res 53, 389-397.

19. Cienfuentes-Jovellanos E, Pasamar MA, Fritz J, et al. (2007) Method for Obtaining Polyphenol-Rich Cocoa Powder with a Low Fat Content and Cocoa thus Obtained. Patent Cooperation Treaty (PCT) WO 2007/096449A1. Spain: Natraceutical Industrial.

20. Ramiro-Puig E, Pérez-Cano FJ, Ramírez-Santana C, et al. (2007) Spleen lymphocyte function modulated by a cocoaenriched diet. Clin Exp Immunol 149, 535-542.

21. Massot-Cladera M, Franch A, Castellote C, et al. (2013) Cocoa flavonoid-enriched diet modulates systemic and intestinal immunoglobulin synthesis in adult Lewis rats. Nutrients $\mathbf{5}$, 3272-3286.

22. Dulloo AG (2011) The search for compounds that stimulate thermogenesis in obesity management: from pharmaceuticals to functional food ingredients. Obes Rev 12, 866-883.

23. Singh A, Holvoet S \& Mercenier A (2011) Dietary polyphenols in the prevention and treatment of allergic diseases. Clin Exp Allergy 41, 1346-1359.

24. Castell M, Pérez-Cano FJ, Abril-Gil M, et al. (2014) Flavonoids on allergy. Curr Pharm Des 20, 972-987.

25. Pérez-Berezo T, Franch A, Castellote C, et al. (2012) Mechanisms involved in down-regulation of intestinal IgA in rats by high cocoa intake. J Nutr Biochem 23, 838-844.

26. Akiyama H, Sato Y, Watanable T, et al. (2005) Dietary unripe apple polyphenols inhibits the development of food allergies in murine models. FEBS Lett 579, 4485-4491.
27. Hara H, Orita N, Hatano S, et al. (1995) Effect of tea polyphenols on fecal flora and fecal metabolic products of pigs. J Vet Med Sci 57, 45-49.

28. Tzounis X, Vulevic J, Kuhnle GG, et al. (2008) Flavanol monomer-induced changes to the human faecal microflora. Br J Nutr 99, 782-792.

29. Bialonska D, Kasimsetty SG, Schrader KK, et al. (2009) The effect of pomegranate (Punica granatum L.) by products and ellagitannins on the growth of human gut bacteria. J Agric Food Chem 57, 8344-8349.

30. Cueva C, Sánchez-Patán F, Monagas M, et al. (2013) In vitro fermentation of grape seed flavan-3-ol fractions by human faecal microbiota: changes in microbial groups and phenolic metabolites. FEMS Microbiol Ecol 83, 792-805.

31. Dolara P, Luceri C, De Filippo C, et al. (2005) Red wine polyphenols influence carcinogenesis, intestinal microflora, oxidative damage and gene expression profiles of colonic mucosa in F344 rats. Mutat Res 591, 237-246.

32. Sembries S, Dongowski G, Mehrländer K, et al. (2006) Physiological effects of extraction juices from apple, grape, and red beet pomaces in rats. J Agric Food Chem 54, 10269-10280.

33. Selma MV, Espín JC \& Tomás-Barberán FA (2009) Interaction between phenolics and gut microbiota: role in human health. J Agric Food Chem 57, 6485-6501.

34. Puupponen-Pimiä R, Nohynek L, Hartmann-Schmidlin S, et al. (2005) Berry phenolics selectively inhibit the growth of intestinal pathogens. J Appl Microbiol 98, 991-1000.

35. Duda-Chodak A (2012) The inhibitory effect of polyphenols on human gut microbiota. J Physiol Pharmacol 63, 497-503.

36. Daims H, Brühl A, Amann R, et al. (1999) The domainspecific probe EUB338 is insufficient for the detection of all bacteria: development and evaluation of a more comprehensive probe set. Syst Appl Microbiol 22, 434-444.

37. Manz W, Amann R, Ludwig W, et al. (1996) Application of a suite of $16 \mathrm{~S}$ rRNA-specific oligonucleotide probes designed to investigate bacteria of the phylum Cytophaga-Flavobacter-Bacteroides in the natural environment. Microbiology 142, 1097-1106.

38. Langendijk PS, Schut F, Jansen GJ, et al. (1995) Quantitative fluorescent in situ hybridisation of Bifidobacterium spp. with genus specific $16 \mathrm{~S}$ rRNA targeted probes and its application in fecal samples. Appl Environ Micriobiol 61, 3069-3075.

39. Harmsen HJM, Elfferich P, Schut F, et al. (1999) A $16 \mathrm{~S}$ rRNAtargeted probe for detection of lactobacilli and enterococci in faecal samples by fluorescent in situ hybridization. Microb Ecol Health Dis 11, 3-12.

40. Poulsen LK, Lan F, Kristensen CS, et al. (1994) Free in PMC spatial distribution of Escherichia coli in the mouse large intestine inferred from rRNA in situ hybridization. Infect Immun 62, 5191-5194

41. Franks AH, Harmsen HJM, Raangs GC, et al. (1995) Variations of bacterial populations in human feces measured by fluorescent in situ hybridization with group specific 16S rRNA-targeted oligonucleotide probes. Appl Environ Microbiol 64, 3336-3345.

42. Trebesius K, Leitritz L, Adler K, et al. (2000) Culture independent and rapid identification of bacterial pathogens in necrotizing fasciitis and streptococcal toxic shock syndrome by fluorescence in situ hybridisation. Med Microbiol Immunol 188, 169-175.

43. Wallner G, Amann R \& Beisket W (1993) Optimizing fluorescent in situ hybridization with rRNA-targeted oligonucleotide probes for flow cytometric identification of microorganisms. Cytometry 14, 136-143. 